\title{
Subjective easiness of pain assessment measures in older people
}

\section{Rapo-Pylkkö, Susanna}

2016-07-01

Rapo-Pylkkö , S , Haanpaa , M \& Liira , H 2016 , ' Subjective easiness of pain assessment measures in older people ' , Archives of Gerontology and Geriatrics , vol. 65 , pp. 25-28 . https://doi.org/10.1016/j.arc

http://hdl.handle.net/10138/224010

https://doi.org/10.1016/j.archger.2016.02.011

publishedVersion

Downloaded from Helda, University of Helsinki institutional repository.

This is an electronic reprint of the original article.

This reprint may differ from the original in pagination and typographic detail.

Please cite the original version. 


\title{
Subjective easiness of pain assessment measures in older people
}

\author{
Susanna Rapo-Pylkköa,b,*, Maija Haanpääc,d, Helena Liira ${ }^{\mathrm{b}, \mathrm{e}}$ \\ a Kirkkonummi Health Center, Kirkkonummi, Finland \\ ${ }^{\mathrm{b}}$ Unit of Primary Health Care, Helsinki University Central Hospital and University of Helsinki, Helsinki, Finland \\ ${ }^{\mathrm{c}}$ Mutual Insurance Company Etera, Helsinki, Finland \\ d Department of Neurosurgery, Helsinki University Hospital, Helsinki, Finland \\ e General Practice, School of Primary, Aboriginal and Rural Health Care, University of Western Australia, Crawley, Australia
}

\section{A R T I C L E I N F O}

\section{Article history:}

Received 2 November 2015

Received in revised form 15 January 2016

Accepted 15 February 2016

Available online 17 February 2016

\section{Keywords:}

Chronic pain

Community-dwelling

Assessment

Self-report

\begin{abstract}
A B S T R A C T
Background: Chronic pain is a significant health problem in older people. Easy-to-use measures of pain help detection and assessment of pain in general practice.

Objective: To assess the subjective ease of use of self-report tools of pain among older home-dwelling people.

Methods: Independently living people aged 75, 80 and 85 years subject to communal preventive home visits with chronic pain were invited to a clinical pain examination. At the visit subjects were asked to assess their pain with 4 self-report tools; VAS (visual analog scale), NRS (numerical rating scale), PainDETECT and BPI (brief pain inventory), and at the end report the subjective ease of use for these measures.

Results: Altogether 106 subjects (28 males, 78 females) consented to participate in the clinical study. Musculoskeletal pain was the most common cause of chronic nociceptive pain, being present in 88 ( $83 \%$ ) subjects. The most common manifestations were spinal disorders and osteoarthritis of the hip or knee. The multidimensional pain scales (PainDETECT and BPI) were rated easier to use than the unidimensional measures (VAS and NRS), although all the measures were assessed as "quite easy" to use in general. In comparison with the other measures, PainDETECT was significantly easier to use. Low MMSE explained the difficulty of using PainDETECT.

Conclusions: The VAS, NRS, PainDETECT and the BPI appear to be suitable pain measures to use for older community-dwelling people in general practice.
\end{abstract}

(c) 2016 Published by Elsevier Ireland Ltd.

\section{Introduction}

Chronic pain is common in the general population, and its prevalence increases with age (Gibson \& Lussier, 2012). Easy-touse measures of pain are needed in clinical contexts and in research. Assessing levels of pain can be challenging in older people, since the measures may be difficult for some of them to understand. If that is the case, the validity and reliability of measurements decrease.

Self-report tools are an important component of pain assessment (Herr \& Garand, 2001). Pain intensity may be measured with an 11-point numerical rating scale (NRS) $(0=$ no pain, $10=$ "worst possible pain"), a visual analog scale (VAS) or a verbal rating scale (Jensen \& Karoly, 2001). The VAS and NRS are recommended for

\footnotetext{
* Corresponding author at: Kirkkonummi Health Center, Virkatie 1, 02400 Kirkkonummi, Finland.

E-mail address: susanna.rapo-pylkko@kirkkonummi.fi (S. Rapo-Pylkkö).
}

both daily practice and clinical trials (Dworkin et al., 2005; Haanpää et al., 2011). They are regarded as suitable for cognitively intact older people who are capable of self-report (Breivik et al., 2008; Hadjistavropoulos et al., 2007; Tiplady, Jackson, Maskrey, Swift, 1998). The NRS is supposed be easier to use than the VAS for elderly people (Dworkin et al., 2005). It is also regarded as the most reliable for assessing treatment effects in the case of chronic pain (Dworkin et al., 2005).

When assessing chronic pain, it is important to also measure subjective disability, i.e., the limitations on functioning associated with pain (Hadjistavropoulos et al., 2007). A generic measure for this is the Interference subscale of the Brief Pain Inventory (BPI) (Cleeland \& Ryan, 1994). It is recommended for use with different pain conditions, whereas condition-specific measures are recommended for specific disease entities (e.g., Oswestry Disability Index for patients with low back pain).

It is important to recognize neuropathic pain, i.e., pain caused by a lesion or disease of the somatosensory system (www.iasppain.org), in older people, as many of its causes are more prevalent 
among older people. In recent years, several screening tools have been developed and validated for neuropathic pain (Bennett et al., 2007). Among them, PainDETECT is available also in Finnish. It was originally developed to detect a neuropathic pain component in low back pain (Freynhagen, Baron, Gockel, \& Tölle, 2006), but has also been validated for other neuropathic pain conditions.

In this study we report on subjective ease of use of the VAS, NRS, and PainDETECT, as well as the Intensity and Interference subscales of the BPI among older home-dwelling people.

\section{Materials and methods}

\subsection{Subjects}

The subjects were recruited into the study during preventive home visits organized by the municipality of Kirkkonummi (population, 37,600 inhabitants in 2012 in Southern Finland) for older people aged 75,80 and 85 years who were living independently at home in the period 2009-2013. We recruited population-based study cohorts from three age groups: 75-yearolds (born 1933-1935), 80-year-olds (born 1931-1932) and 85-year-olds (born 1924-25), and gave them the opportunity to participate in the current study. The target age group consisted of 802 elderly patients, 684 of whom lived independently at home. Of them, 460 consented to participate in the preventive home visits, and 175 (38\%) fulfilled the inclusion criteria of the current study. Altogether 106 patients (28 males, 78 females, 26\% and 74\%, respectively) consented to participate in the clinical study.

Among other things the home visit included MMSE testing and pain evaluation using a one-sheet questionnaire. It inquired about the presence of chronic (duration $>3$ months) pain and its intensity. Those experiencing pain with an average daily intensity of $>4$ on an NRS during the previous week or with at least moderate interference in daily life were offered a consultation with a geriatrician (SR-P). The exclusion criteria were impaired cognitive function (MMSE $<23$ ) or impaired communication skills.

\subsection{Methods}

At the beginning of the visit the study nurse gave a blank body chart to the subject and asked him or her to draw on any areas of pain. After that the subject was asked to assess the intensity of the pain with the VAS. In the cases of more than one different pain states the worst pain state was assessed. The maximum intensity and the average intensity of the pain during the last week were assessed separately. Then the subject was asked to fill in the Severity and Intensity subscales of the BPI and PainDETECT.

The BPI is a patient-completed numeric rating scale that assesses the severity of pain (severity scale) and its impact on daily functioning (interference scale). The Pain Interference Scale assesses the degree to which pain interferes with seven daily activities (general activity, mood, normal work, walking, relations with others, sleep, and enjoyment of life). The ratings are measured using 11-point numeric rating scales ranging from 0 (does not interfere) to 10 (completely interferes). The mean of these seven ratings is used to indicate the patient's overall level of pain interference.

PainDETECT contains 9 items. There are 7 weighted sensory descriptor items and 2 items relating to the spatial (radiating) and temporal characteristics of the individual pain pattern.

After being interviewed by the nurse, the subject was examined by the geriatrician. A review of the recent medical history included an assessment with an NRS of the intensity of the worst and average pain during the previous week. The clinical examination aimed at diagnosing the etiology of the pain state(s) and the type (s) of pain (nociceptive, neuropathic, combination of both). At the end of the visit the subjects were asked to assess the ease of use for the VAS, NRS, BPI and PainDETECT using a 7-point verbal rating scale ( $1=$ very easy, 2 =easy, $3=$ quite easy, $4=$ not easy, not difficult, 5 =quite difficult, 6 = difficult, 7 = very difficult).

\subsection{Statistics}

The within-subject measures were analyzed using generalizing estimating equation (GEE) models with an unstructured correlation structure. Generalized estimating equations were developed as an extension of the general linear model (e.g., OLS regression analysis) to analyze longitudinal and other correlated data. GEE models take into account the correlation between repeated measurements in the same subject; models do not require complete data and can be fit even when individuals do not have observations at all time points. Multivariate linear regression analyses were used to identify the appropriate predictors of ease of using the measures using the standardized regression coefficients beta $(\beta)$. The beta value is a measure of how strongly each predictor variable influences the criterion (dependent) variable. The beta is measured in units of standard deviation. Cohen's standard for Beta values above $0.10,0.30$ and 0.50 represent small, moderate and large relationships, respectively. Hochberg's procedure and Sidak's adjustment were used to correct type I error. The bootstrap method was used when the theoretical distribution of the test statistics were unknown or in the event of a violation of the assumptions (e.g., non-normality). Partial correlations were calculated between ease of use of the measures, adjusted for age, gender and MMSE.

The study protocol was approved by the Ethics Committee of the Helsinki University Central Hospital (permission 128/13/03/ $00 / 09$ ), and written informed consent was obtained from all participants.

\section{Results}

Altogether 106 subjects (28 males, 78 females) consented to participate in the clinical study. Of them, 65 patients were 75 years old, 28 were 80 years old and 13 were 85 years old. Mean intensity (SD) of pain on the NRS scale during the previous week was 5.7 (1.6), and the intensity of maximal pain was 7.7 (1.6).

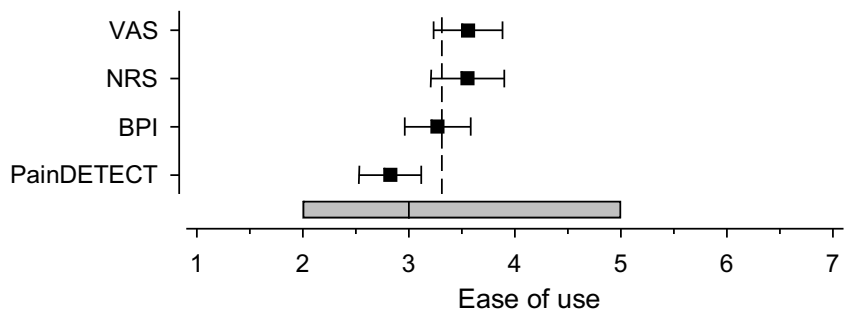

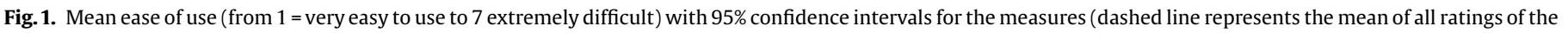
measures, and the box-plot represents medians and inter-quartile ranges). 
Table 1

Multiple regression analysis for ease of use of the measures.

\begin{tabular}{|c|c|c|c|c|}
\hline & VAS beta, $\beta(95 \% \mathrm{CI})$ & NRS beta, $\beta(95 \% \mathrm{CI})$ & BPI interference scale beta, $\beta$ (95\% CI) & $\begin{array}{l}\text { PainDETECT } \\
\text { beta, } \beta(95 \% \mathrm{CI})\end{array}$ \\
\hline Female gender & $0.13(-0.07$ to 0.34$)$ & $0.14(-0.07$ to 0.36$)$ & $0.10(-0.10$ to 0.30$)$ & $0.12(-0.07$ to 0.32$)$ \\
\hline \multicolumn{5}{|l|}{ Age } \\
\hline 75 & Reference & Reference* & Reference & Reference \\
\hline 80 & $0.00(-0.20$ to 0.20$)$ & $0.12(-0.08$ to 0.33$)$ & $0.12(-0.08$ to 0.31$)$ & $-0.01(-0.21$ to 0.19$)$ \\
\hline 85 & $0.15(-0.05$ to 0.34$)$ & $0.26(0.06$ to 0.46$)$ & $0.16(-0.03$ to 0.36$)$ & $0.06(-0.14$ to 0.26$)$ \\
\hline \multicolumn{5}{|l|}{ Type of pain } \\
\hline No neuropathic pain & Reference & Reference & Reference & Reference \\
\hline Neuropathic pain present & $-0.21(-0.41 \text { to }-0.02)^{* *}$ & $0.03(-0.19$ to 0.25$)$ & $0.04(-0.17$ to 0.24$)$ & $-0.05(-0.27$ to 0.18$)$ \\
\hline MMSE result & $-0.10(-0.29$ to 0.10$)$ & $0.05(-0.16$ to 0.25$)$ & $-0.13(-0.32$ to 0.06$)$ & $-0.20(-0.39 \text { to }-0.01)^{* * * *}$ \\
\hline Results of the scale & $0.00(-0.20$ to 0.20$)$ & $-0.03(-0.24$ to 0.18$)$ & $0.19(0.01-0.38)^{* * * *}$ & $-0.13(-0.34$ to 0.09$)$ \\
\hline
\end{tabular}

Musculoskeletal pain was the most common cause of nociceptive pain, being present in $88(83 \%)$ subjects. The most common manifestations of it were spinal disorders (48 subjects) and osteoarthritis of the hip or knee (22 subjects). Neuropathic pain was diagnosed in 51 (48\%) subjects. The most common cause for neuropathic pain was radiculopathy caused by a degenerative disease of the spinal column (36 subjects). Neuropathic pain was caused by peripheral nerve trauma in 8 and by peripheral nerve entrapment in 7 subjects.

The NRS was difficult or very difficult to use for $46 \%$ of the subjects, and $36 \%$ in regard to the VAS, whereas PainDETECT and BPI were regarded as difficult or very difficult to use by $23 \%$ and $31 \%$, respectively.

Subjective ease of use of the measures is presented in Fig. 1. There was a significant difference between the measures $(p<0.001$ after adjusting for age and gender). PainDETECT was regarded as the easiest to use. In comparison with the other measures, PainDETECT was significantly easier to use (in comparison with the VAS and with NRS $p<0.001$ and in comparison with the BPI $p=0.009$ ). The intraclass correlation between ease of use of the measures was 0.84 (95\% CI: 0.78-0.89).

Table 1 presents the variables that explain the difficulties in using each measure. The presence of neuropathic pain was associated with ease of use of the VAS $(p=0.032)$. Age explained the difficulty of using the NRS $(p=0.0018)$. A high score in the Interference subscale rating was associated with difficulty in using the BPI $(p=0.049)$. Low MMSE explained the difficulty of using PainDETECT.

The correlations between the ease of use of the measures (after adjusting for MMSE result, age and gender) are presented in Table 2. Adjusted correlations varied from 0.49 to 0.73 , with the strongest correlation $(0.73)$ found between difficulty of using the NRS and the BPI (interference subscale).

Table 2

Correlation between subjective ease of use of the measures after adjusting for MMSE result, age and gender.

\begin{tabular}{llll}
\hline & VAS & NRS & BPI inter \\
\hline NRS & $0.53(0.31-0.72)^{* * *}$ & & \\
BPI inter & $0.60(0.40-0.75)^{* * *}$ & $0.73(0.58-0.83)^{* * *}$ & \\
PainDETECT & $0.49(0.28-0.65)^{* * *}$ & $0.55(0.33-0.69)^{* * * *}$ & $0.54(0.34-0.70)^{* * *}$ \\
\hline
\end{tabular}

Sidak-adjusted probabilities: ${ }^{*} p<0.05,{ }^{* *} p<0.01,{ }^{* * *} p<0.001$.

\section{Discussion}

In our study, the subjective ease of use of four pain measures was evaluated. In general, all of them were assessed as "quite easy" to use on average. However, the subjects rated the use of the multidimensional scales (PainDETECT and BPI) as easier than the unidimensional measures (VAS and NRS). The quick and simple NRS and VAS are suitable for measuring acute pain, such as postsurgical pain (Gagliese \& Katz, 2003), but they do not cover the multiple dimensions of chronic pain (Doventas et al., 2011). Although PainDETECT contains nine questions, it appeared to be user-friendly and easy to comprehend. However, using it was more difficult when the patient's MMSE result deteriorated. The questions from the BPI Interference scale covered broadly various dimensions of function, and in spite of having seven items, it was regarded as relatively easy to use. However, the higher the rating was, the more difficult it was to use.

This study did not assess the reliability or validity of the measures but evaluated only their subjective ease of use. The measures were used on the same visit, which made a comparison of them possible. The patient cohort was recruited from the general population and included a broad spectrum of various pain states, providing a quite representative sample of older people with pain in primary health care. The proportion of males was small (26\%), and only a minority (12\%) of patients belonged to the oldest age group ( 85 years), most likely due to the inclusion criteria (independent living at home and with good cognition). This limits the generalization of the results.

\section{Conclusion}

The VAS, NRS, PainDETECT, and the Interference subscale of the BPI were easy to use, and the assessment of ease of use showed high intraclass correlation in this cohort. These measures appear to be suitable for elderly patients in general practice.

\section{Conflicts of interest}

There were no conflicts of interest.

\section{Funding}

The study received financial support from the research fund of Orton. Writers appreciate their support for this study. 


\section{Ethical approval}

All procedures performed in studies involving human participants were in accordance with ethical standards of the institutional research committee and with the 1964Helsinki declaration and its later amendments or comparable ethical standards. The study protocol was approved by the Ethics Committee of the Helsinki University Central Hospital (permission $128 / 13 / 03 / 00 / 09$ ), and written informed consent was obtained from all participants.

\section{References}

Bennett, M. I., Attal, N., Backonja, M. M., Baron, R., Bouhassira, D., Freynhagen, R., et al. (2007). Using screening tools to identify neuropathic pain. Pain, 127,199-203.

Breivik, H., Borchgrevink, P. C., Allen, S. M., Rosseland, L. A., Romundstad, L., Hals, E. K., et al. (2008). Assessment of pain. British Journal of Anaesthesia, 101(1), 17-24. Cleeland, C. R., \& Ryan, K. M. (1994). Pain assessment: global use of the brief pain inventory. Annals of the Academy of Medicine, 23, 130-138.

Doventas, A., Karadag, B., Curgunlu, A., Bilici, A., Sut, N., Erdincler, D. S., et al. (2011). Replicability and reliability of pain assessment forms in geriatrics. Archives of Gerontology and Geriatrics, 53, 55-60.
Dworkin, R. H., Turk, D. C., Farrar, J. T., Haythornthwaite, J. A., Jensen, M. P., Katz, N. P., et al. (2005). Core outcome measures for chronic pain clinical trials: IMMPACT recommendations. Pain, 113, 9-19.

Freynhagen, R., Baron, R., Gockel, U., \& Tölle, T. R. (2006). PainDETECT: a new screening questionnaire to identify neuropathic components in patients with back pain. Current Medical Research and Opinion, 22, 1911-1920.

Gagliese, L. \& Katz, J. (2003). Age differences in postoperative pain are scale dependent: a comparison of measures of pain intensity and quality in younger and older surgical patients. Pain, 103, 11-20.

Gibson, D. J., \& Lussier, D. (2012). Prevalence and relevance of pain in older persons. Pain Medicine, 13, 23-26.

Haanpää, M., Attal, N., Backonja, M., Baron, R., Bennett, M., Bouhassira, D., et al. (2011). NeuPSIG guidelines on neuropathic pain assessment. Pain, 152, 14-27.

Hadjistavropoulos, T., Herr, K., Turk, D. C., Fine, P. G., Dworkin, R. H., \& Helme, R. (2007). An interdisciplinary expert consensus statement on assessment of pain in older persons. The Clinical Journal of Pain, 23, S1-S43.

Herr, K. A., \& Garand, L. (2001). Assessment and measurement of pain in older adults. Clinics in Geriatric Medicine 17(3), 457-478. http://www.iasp-pain.org/ Taxonomy? navitemNumber=576\#Peripheralneuropathicpain.

Jensen, M. P., \& Karoly, P. (2001). Self-report scales and procedures for assessing pain in adults. In D. C. Turk, \& R. Melzack (Eds.), Handbook of pain assessment (pp. 135-151).New York: Guilford Press.

Tiplady, B., Jackson, S., Maskrey, V., \& Swift, C. (1998). Validity and sensitivity of visual analogue scales in young and older healthy subjects. Age and Ageing, 27, $63-66$. 